\title{
Mathematical Modelling of the Response of Breast Cancer to Drug Therapy
}

\author{
D. A. CAMERON* \\ Directorate of Oncology, Westem General Hospital, Edinburgh, Scotland
}

(Received 24 January 1997)

\begin{abstract}
Mathematical modelling of tumour response in breast cancer offers the potential for further understanding of the mechanisms involved in a tumour's imperfect response to chemotherapy. Three different models of assessing response are studied; the simplest consisting of fitting a regression line to the logarithm of the tumour volumes; a study using exponential growth and an S-shaped growth response curve; and one that assumes $\log$ cell-kill and the possibility of primary tumour resistance to therapy. All three can explain some facets of tumour biology, but it is the introduction of the possibility of resistance that appears to result in correlations with clinical outcome. The issue of Gompertz growth is discussed, since it is considered, although not without controversy, to best describe not only xenograft but also clinical tumour growth, and yet has not been used in any of the three models discussed. It appears that much of the data used to clinically validate Gompertz growth is before the period of maximum deceleration, and thus the true relevance of this function to clinical tumour growth remains uncertain.
\end{abstract}

Keywords: breast cancer, chemotherapy, gompertz, growth modelling, response, resistance

\section{INTRODUCTION}

The treatment of breast cancer with cytotoxic drugs remains a challenge to the clinician, despite recent advances in the understanding of the biology of the disease and the increased armamentarium of available drugs. In the majority of instances, breast cancer is sensitive to drug therapy, but rarely cured by it. There are many reasons for the lack of complete sensitivity to treatment, but a better understanding of the manner in which tumours respond to treatment might help design more effective therapies, and a mathematical approach to the pattern of response could therefore shed light on this problem.

Chemotherapy is widely used in the treatment of breast cancer. Following definitive locoregional treatment, usually comprising surgery and radiotherapy, it has been clearly shown that several months' of cytotoxic drug therapy improves the long-term survival (Early Breast Cancer Trialists Collaborative Group, 1992). This is because many such women have microscopic residual disease, often widely disseminated. However the survival curves rarely plateau, and this has been interpreted as an indicator

\footnotetext{
${ }^{*}$ Correspondence to: Dr. David Cameron, Department of Oncology, Western General Hospital, Crewe Road South, EDINBURGH EH4 2XU
} 
that the main effect of the drug therapy is cytoreductive; women with residual disease will still relapse from their disease, but the effect of the drug therapy has been to reduce the tumour burden and thus delay the time to relapse and death (Henderson, 1995). However since the chemotherapy is given in the absence of definite disease, there is no possibility of observing the tumour response, and thus the benefit for an individual can only be inferred from the improved survival of the group.

There are however a significant number of women who present with disease that is either overtly metastatic, or too locally advanced for surgery. Primary therapy with systemic cytotoxic drug therapy is indicated - for palliation in the first instance and to improve local (and possibly distant) control of the disease in the latter. In these patients the response of the tumour to the applied therapy can be observed, and it is not surprising that response to the therapy is not only associated with better symptom palliation but also, in some circumstances, prolonged survival. Since the drugs used are similar, this setting can be used to assay the efficacy of new treatments, and also for biological studies of tumour response to therapy. Indeed the successful cyto-reduction of inoperable locally advanced breast cancer by primary systemic therapy can render tumours operable, and has therefore led to a possible revolution in the management of early (operable) breast cancer (Epstein, 1996). Since there are data to suggest that surgical removal of the primary tumour can accelerate the growth of distant disease (Fisher $e t$ al 1983), it could be beneficial to the patient to have her systemic drug therapy before surgery. Based in part on the response of locally advanced disease to systemic drug therapy, there have been a number of studies of such pre-operative systemic therapy to determine the efficacy and potential survival advantage of this approach. Although none of the randomised studies have reported an overall clear survival advantage (Mauriac et al 1991; Powles et al 1995; Scholl et al 1995; Scholl et al 1994; Semiglazov et al 1994), response to such therapy is associated with a better outcome (Calais et al 1993; Cameron et al 1995; Jacquillat et al 1990;
Scholl et al 1996). However the precise factors that predict for response, and in particular longterm survival, remain unclear; particularly as one of the few biological predictors for a good response to chemotherapy, namely the proliferative fraction of the tumour (Chevillard et al 1996; Gardin et al 1994; Remvikos et al 1993a), is at the same time a predictor for a poorer long-term survival (Clayton, 1991; Gasparini et al 1994). The apparent discrepancy between these two is perhaps in part explained by the observation that it is in the non-responsive tumours that the high proliferative fraction predicts for a particularly poor outcome (Remvikos et al 1993b). Another factor associated with resistance to chemotherapy, P-glycoprotein expression (Harris et al 1992), has been shown to predict for a lack of response to pre-operative chemotherapy for some regimens but not others (Chevillard et al 1996).

It is clear from the many studies conducted that most patients still relapse and die after apparently effective drug therapy; whether in the metastatic, locally advanced or preoperative setting. Since tumour response is associated with a survival advantage, it might be possible to model the pattern of response mathematically in order to gain insight into the relative importance of the constituent parts of a tumour's response to therapy, and thus improve the design of the regimens.

However any attempt to model tumour response must incorporate at least the possibility of tumour re-growth during therapy, and therefore a brief introduction to three commonly considered models of tumour growth is necessary. The simplest is that of exponential growth, where there is a constant growth rate $\alpha$, and the volume $W$ (or cell number) is given by:

$$
W=W_{0} \exp (\alpha t)
$$

(where $\boldsymbol{W}_{0}$ is the initial volume, and $\alpha$ the growth rate).

Clinical experience suggests that this does not give an accurate description of tumour growth during its entire history from a single cell until death of the host, in particular as it does not allow for any growth decceleration. Skehan (1984) showed that all metazoan growth is ultimately deccelerating, and 
therefore two models of bounded growth need also to be considered. The Gompertz curve, first published in 1825 (Gompertz, 1825), was for a long time only of interest to actuaries, as it modelled the human struggle with mortality using an exponentially decreasing ability to avoid death. It will be discussed in greater detail later (see page 10), but mathematically the volume $W$ is defined as:

$$
W=W_{\infty} \exp \{(\beta / \alpha) \exp (-\alpha t)\}
$$

(where $\boldsymbol{W}_{\infty}$ is the maximum volume, $\beta$ the relative growth rate and $\alpha$ the rate of decay of $\beta$ ).

In this century it has also been used to model both biological and economic growth, and an early review contrasted it with another bounded growth function, the logistic curve (Winsor, 1932). This was first proposed by Verhulst shortly after the Gompertz function (Verhulst, 1838), and is given by the equation:

$$
W=W_{\infty}\left[1+\exp \left(\frac{r}{W_{\infty}}-r t\right)\right]^{-1}
$$

(where $W$ is the volume at time $t, W_{\infty}$ is the maximum volume, and $\boldsymbol{r}$ is the growth rate).

By letting the value of the exponent $(-1$ in above) vary as a variable $I / N$, one has the generalised logistic equation, which was used by Spratt (1993) and will be discussed later (see page 11). For $N>1$, the curve approximates exponential growth, but with a late, rapid decceleration, whereas when
$0<N<1$, the deccelaration becomes ever more pronounced.

\section{CLINICAL STUDIES}

\section{S-shaped Drug Response/Exponential Growth}

The first published model of the response of breast cancer appears to be a series of patients treated with 5-fluorouracil and 6-mercaptopurine in a drug evaluation study (Priore, 1966). The traditional definition of response is based on the difference between the pre-treatment tumour size and the final (or best) tumour size - and because changes in tumour size are a consequence of tumour growth rates as well as drug action, it was felt that there might be better methods for quantifying tumour response. There had already been a number of studies examining tumour growth patterns, with two reporting that exponential growth was adequate to describe the growth of lung tumours of non-breast origin (Collins et al 1956; Schwartz, 1961), and one confirming its applicability to primary breast cancer (Ingleby et al 1958). Other studies had determined the relationship between drug dosage and tumour response, and on the basis of these early pharmacokinetic and pharmacokinetic studies, it was felt that an S-shaped log-dose response curve was appropriate.

Hence the following model was built:

\section{assumptions}

exponential growth with growth rate sensitive fraction of the tumour cumulative dose to time $t$ dose-response cell-kill curve

response function

Thus we have:

$$
Y(t)=(1-\varphi P) \exp ^{\delta t}
$$

Therefore, using a logistic dose-response function for $P(t)$, this becomes:

$\delta$

$\varphi$ $x(t)=\log$ (cumulative dose to time $t$ )

$P(x(t))$, such that $1-P$ is the proportion of the untreated tumour volume that survives to $t$. $Y(t)=$ ratio of the number of cells at time $t$ to the number present at time 0

$$
Y(t)=\frac{1-\varphi}{(1+\exp (-\alpha-\beta x))} \exp (\delta t)
$$

(where $\alpha$ and $\beta$ are the parameters of the logistic equation which can describe different dose-response curves, such that the higher the value of $\beta$, the more 


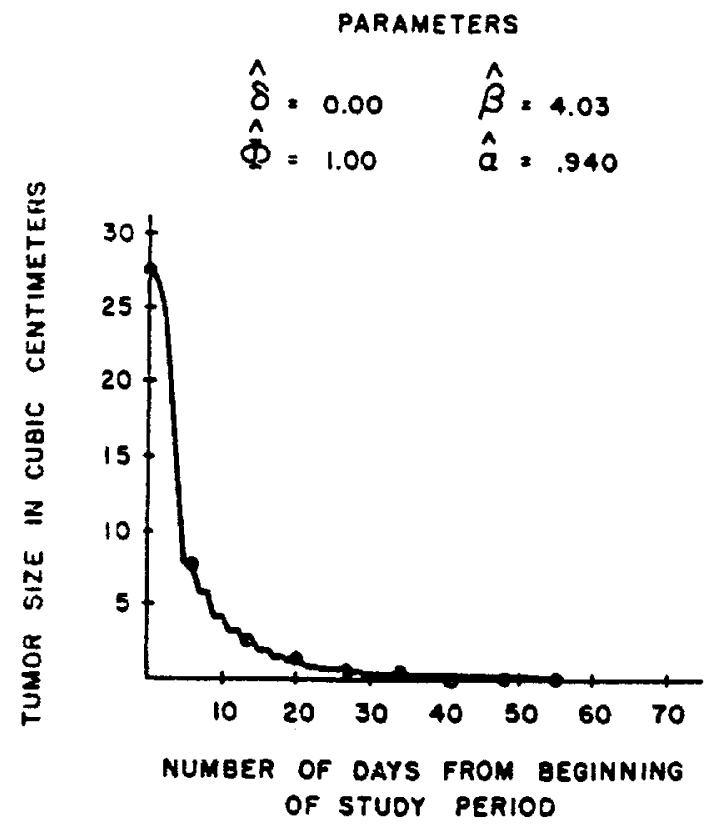

FIGURE 1 An example of low tumour growth rate and high drug cell-kill. The tumour-specific model parameters are shown superscribed with $a \wedge$. The dots represent actual tumour volume measurements, and the bold line the volumes as predicted by the model with the particular parameter values that best fit the volumes. (Reproduced from (Priore, 1966)).

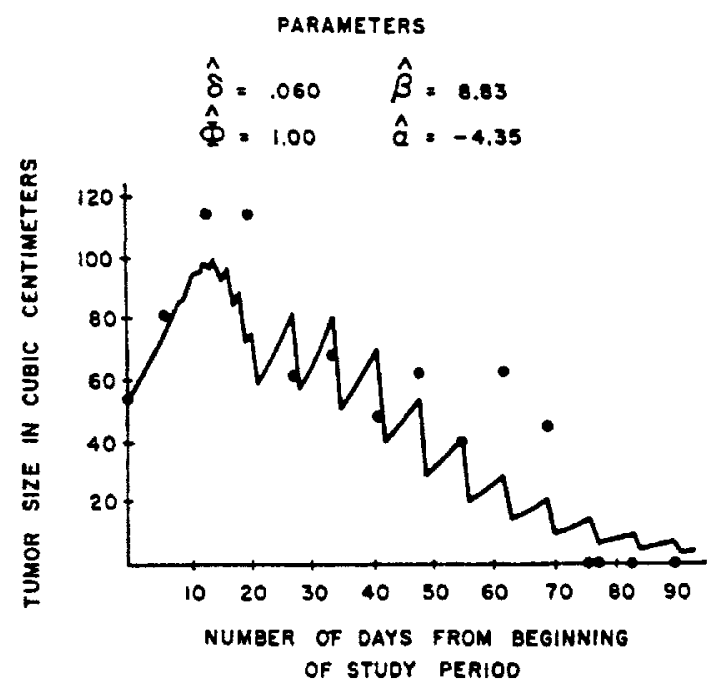

FIGURE 2 An example with high tumour growth rate and high drug cell-kill. The tumour-specific model parameters are shown superscribed with $\wedge$. The dots represent actual tumour volume measurements, and the bold line the volumes as predicted by the model with the particular parameter values that best fit the volumes. This also shows an example of a model detecting tumour regrowth between treatment cycles. (Reproduced from (Priore, 1966)). the drug's effect is concentrated in a narrow time interval, whereas the higher the ratio of $\alpha / \beta$, the earlier the point of maximum drug effect is reached.)

When considering the pattern of clinical tumour response, there are a number of different possibilities, that will depend in part on the underlying growth rate of the tumour, as well as its sensitivity to the therapy. Thus a slow growing tumour may show little change in volume even in the absence of treatment, whereas its faster-growing counterpart will need to be more sensitive to the drug if its volume is to remain unchanged over the course of a few weeks. Even if the drug is very active, the impact will be less immediately obvious in the faster growing tumour, as Figures 1 and 2 illustrate. This may not be a great surprise, but they illustrate figuratively scenarios that are clinically familiar, and Figure 2 shows a phenomenon for which clinical measurements are rarely sufficiently precise -- namely re-growth between treatment courses, of which a further example will be given later (see Figure 7).

Furthermore when considering patients with the same tumour type, it was noted that tumours within one patient were more alike in their behaviour than tumours from different patients, with 4-5 fold differences in these variances. This was particularly true for the drug effect on patients with breast cancer.

It was concluded that this approach could separately identify the roles of tumour growth and drug effects, and indeed was able to determine that one of these drugs, 5-fluorouracil, was significantly more effective than the other for treating colon cancer - and even now 30 years later, single agent 5 -fluorouracil (with or without modulators such as folinic acid) remains the mainstay of treatment of colorectal cancer in both the adjuvant and metastatic settings.

\section{REGRESSION RESPONSE}

Perhaps the simplest model of breast cancer response to be formally studied is that of exponential 
Fastest growing tumour in Heuser's data-set

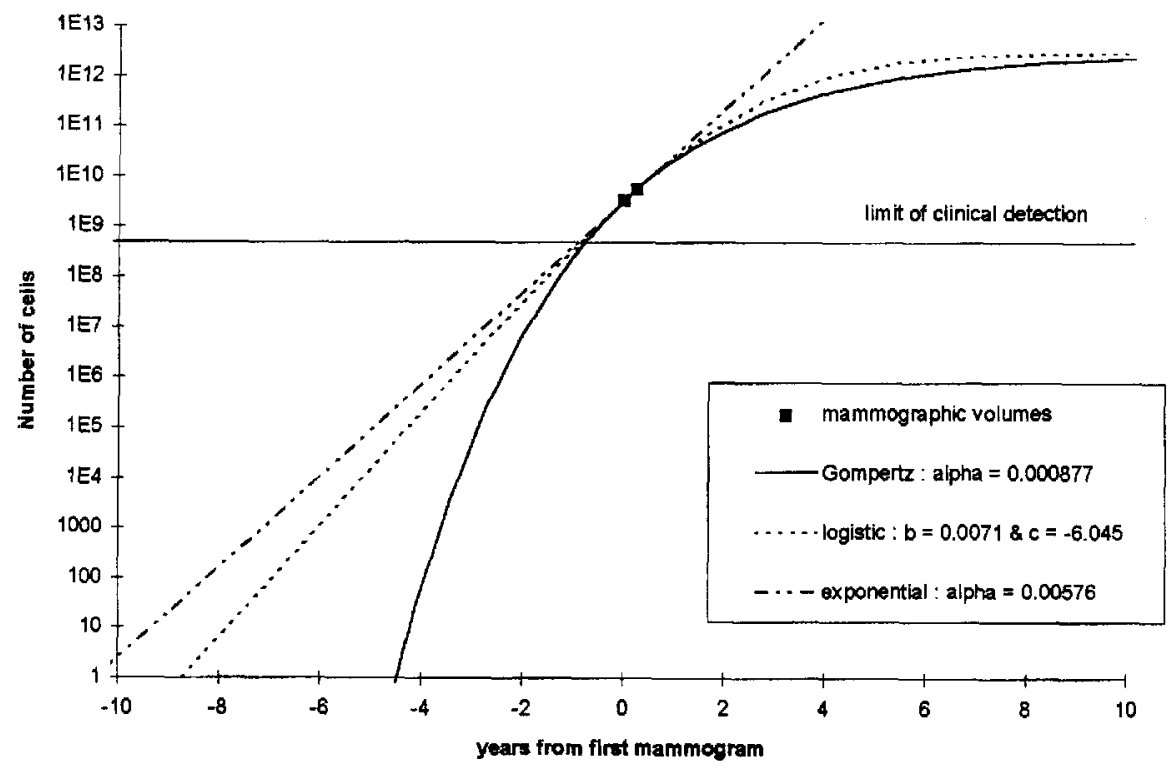

(a)

Slowest growing tumour in Heuser's data-set

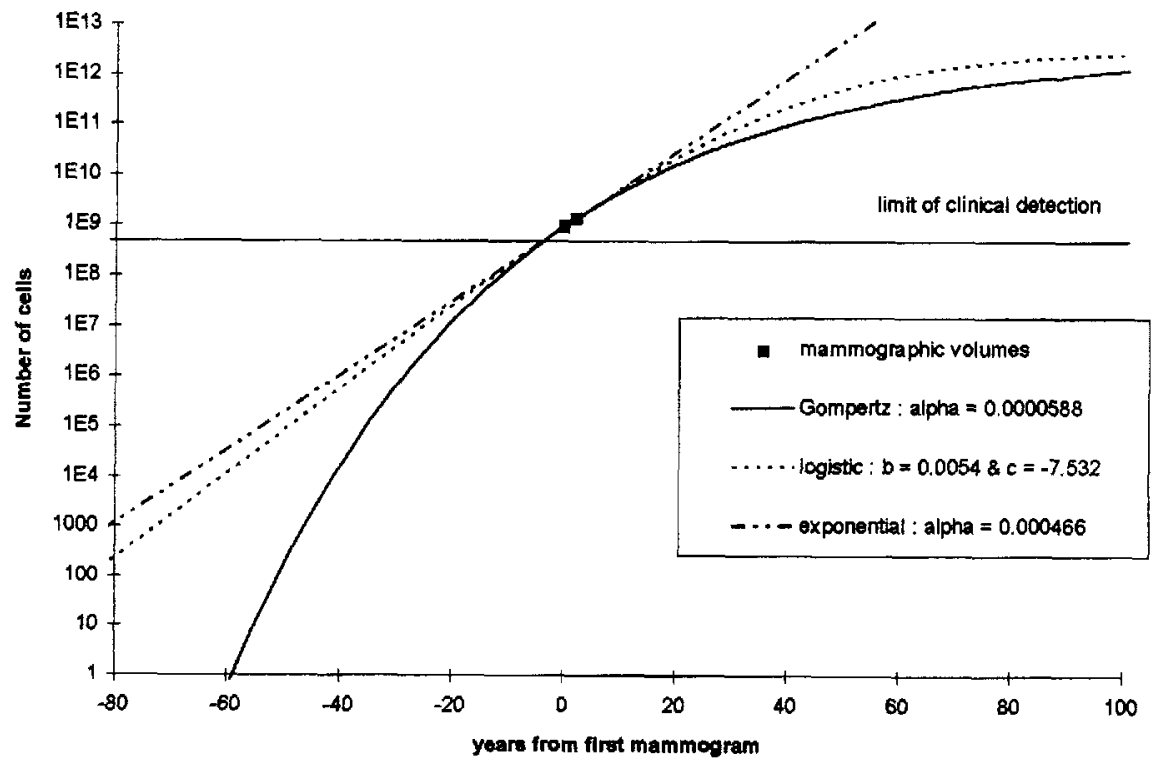

(b)

FIGURE 7 Growth curves - Norton's fit to the Hesuer data set. Both Gompertz and Logistic curves have the same maximum volume, namely $3.1 \times 10^{12}$ cells $(\approx 3$ litres of tumour). 


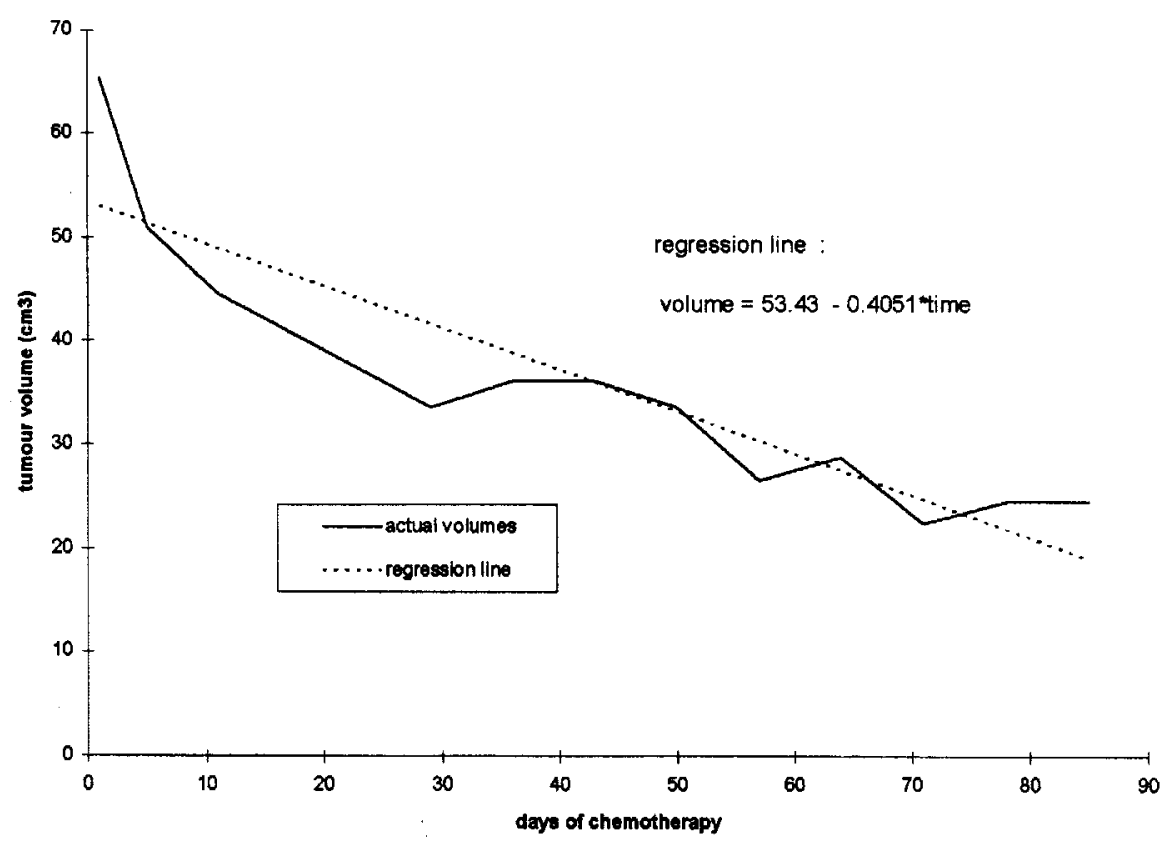

FIGURE 3 An example of a regression line fitted to a tumour responding to chemotherapy: $(t=-6.94, p<0.01)$.

regression. In this model, no particular account is made of tumour growth or resistant cells, but the tumour volumes during treatment are recorded and an attempt is made to fit a linear regression line to their logarithms, giving an exponential regression line (Thomlinson, 1982). Such an approach has been conducted at the Mount Vernon hospital in the UK, and recently data from as far back as 1974 have been reported (Johnson et al 1995). Clinically the approach is attractive for its simplicity, and with only one parameter to be estimated, the use of several data points enhances the confidence of the result. Using the natural logarithms of the tumour volumes, the linear regression capability of any reasonable statistical package can be employed to regress the (log of the) tumours volume on time. Figure 3 gives an example of such a regression line fitted to clinical tumour data.

However although this study (Johnson et al 1995) cannot draw firm conclusions about the power of this approach to determine outcome or even response to therapy, some interesting observations need to be reported. The authors clearly found a correlation between the gradient of the slope and both the grade of the tumour and the treatment applied; with steeper slopes corresponding to higher grade tumours and cytotoxic therapy (administered as chemotherapy or local ionizing radiotherapy). In many cases the authors have fitted a single line to all data points, irrespective of therapy, and with treatment choices not being made upon a simple protocol but rather on the basis of inadequate responses, these conclusions are somewhat undermined. What the authors did conclude was that particularly for grade III tumours, conventional 3-4 weekly cycles of chemotherapy could be inadequate to control the tumour due to re-growth between cycles.

This approach of using regression lines to reflect tumour response was also employed in a phase II study of pre-operative breast cancer for women with large operable primary breast cancer in Edinburgh, commencing in 1984 (Forrest et al 1986). The rationale of the study was to try and improve the survival of women, who by virtue of the size of their tumour, had a relatively poor prognosis despite having early breast cancer. Since the conventional criteria for defining tumour response is a reduction in at least $50 \%$ of the product of two orthogonal diameters 
(Hayward et al 1977), a tumour that regresses more slowly may not be classified as a responder. This situation arises in particular with endocrine therapy, where the tumours may still be sensitive to the applied therapy, but have not regressed a great deal after only 3 months' treatment. However even with chemotherapy the maximum response may be seen after more than 3 months' therapy. Thus it might be felt necessary to change treatment because of apparent resistance, although the tumour is in fact responding but only slowly. Therefore the same approach as Thomlinson was used (1982), with responding tumours defined as those for which the regression of the natural logarithm of the tumour volume (during one type of therapy) against time was significantly down-sloping. It was hoped that this would be a sensitive earlier indicator of tumour response which would predict for long-term benefit (Forrest et al 1986).

We have recently reviewed the survival data on this group of patients, and at the same time also classified response by the UICC criteria of a reduction in area of at least 50\% (Cameron et al 1995). There is however good concordance between the two methods (88\% for chemotherapy and $74 \%$ for endocrine therapy), but for neither group of patients does response as defined by a significant downsloping regression line predict for a survival benefit (Cameron et at 1997), in contrast to the more conventional definition of response, which does result in a survival benefit for patients given chemotherapy (Cameron et al 1995). However for the patients given endocrine therapy, neither method of response definition is associated with a survival benefit. If the rate of regression is considered, as evidenced by the time to halve the tumour volume, then the patients with cancers that respond faster, be it to hormone or chemotherapy, have a better survival (Cameron et al 1997).

Furthermore the gradient of the lines for the 35 patients given chemotherapy are significantly steeper than the 50 given endocrine therapy ( $W=$ 2627, $p<0.0001$ ), confirming the observations of the Mount Vernon group (Johnson et al 1995). There is however no statistical difference between the gradients of the 21 patients given primary chemotherapy, and the 14 patients given the same chemotherapy after failed endocrine therapy.

This all suggests that the approach of using a (log) regression line does reflect the tumour biology, but is not sensitive to all facets of inadequate tumour response, and, in particular, the long-term outcome cannot be predicted by the response of the primary tumour as modeled by regression analysis.

\section{EXPONENTIAL GROWTH/LOG-CELL KILL}

Mindful of the previous attempts to model the response of primary breast cancer to chemotherapy, a composite model was used which had already been successfully applied to the response of smallcell lung cancer (Gregory et al 1990). In this model exponential growth was assumed (as in Priore's original model (Priore, 1966)), each tumour was permitted to contain a proportion that was totally resistant to the applied therapy (also as in Priore's paper), which grew at the same rate as any remaining sensitive tumour cells. The chemotherapy was assumed however to kill a dose-dependent fixed proportion of the sensitive tumour, the so-called logcell kill model (Skipper, 1978) (as in a model of the response of Myeloma to chemotherapy (Sullivan et al 1972) - vide infra). In order to fit the tumour volumes to the model, the method of least likelihood was used, and the entire model written in Microsoft FORTRAN 77. Four tumour volumes were used to derive the model parameters, which could then be used to predict the subsequent tumour volumes. A detailed description of the model is given in appendix 1 .

The first application of this model was to a series of patients with locally advanced breast cancer to whom weekly chemotherapy was given for up to 12 weeks. The details of the chemotherapy and results are given in (Cameron et al 1996), and overall the model gave a good fit to the subsequent tumour regression (as seen for example in Figure 4).

There were a subset of patients who underwent definitive loco-regional surgery after their 


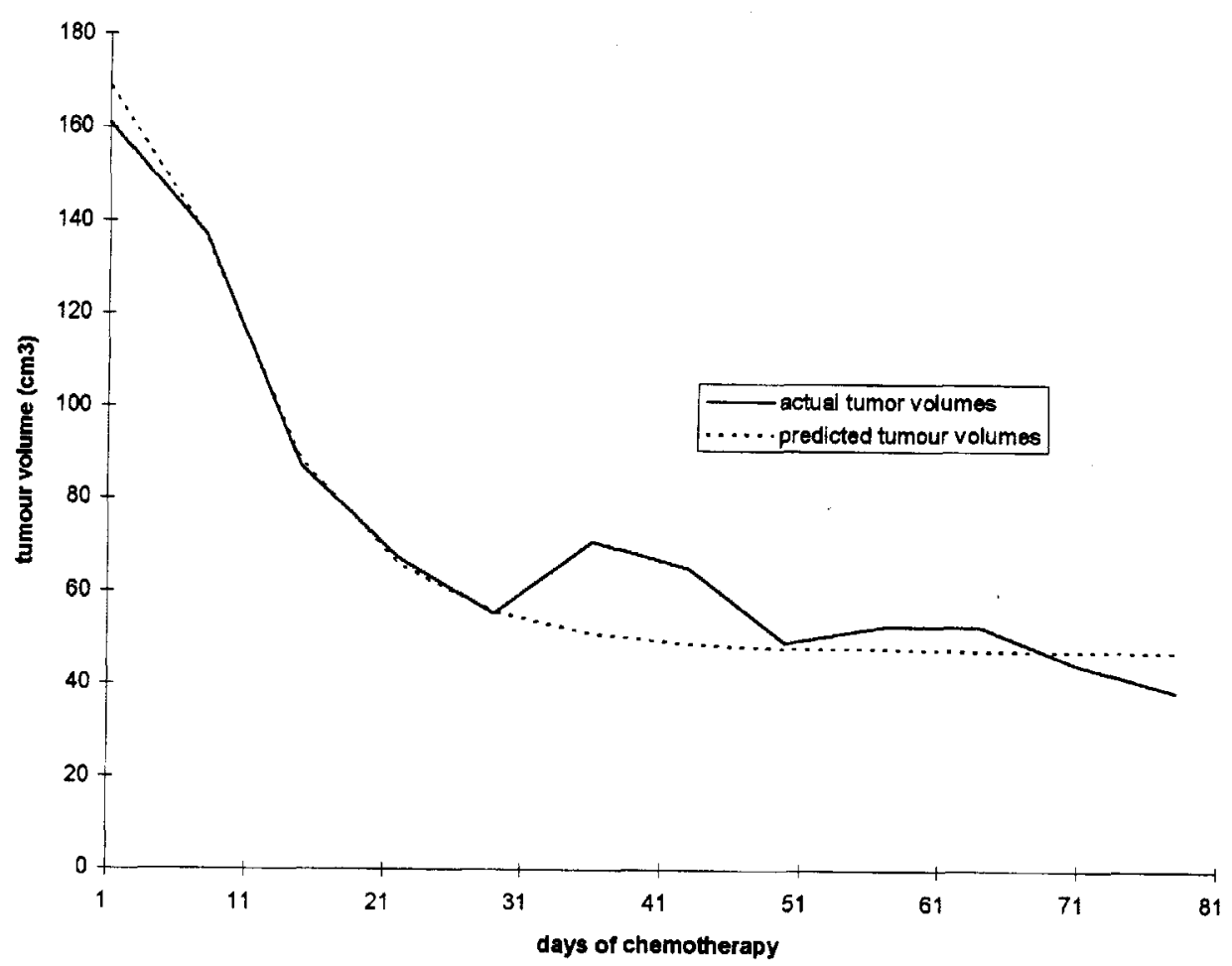

FIGURE 4 An example of a tumour responding to therapy, illustrating the fit of the model growth curve.

chemotherapy, and thus in whom pathological tumour measurements were available. Table I shows the final clinical and pathological volumes for these patients as well as the final volume predicted by the model. Firstly it should be noted that all those with no residual invasive cancer had no initially resistant tumour, as identified by the model. It can further be seen that the final volume as predicted by the model

TABLE I Cell-kinetic parameters and pathological volumes for those tumours having surgery

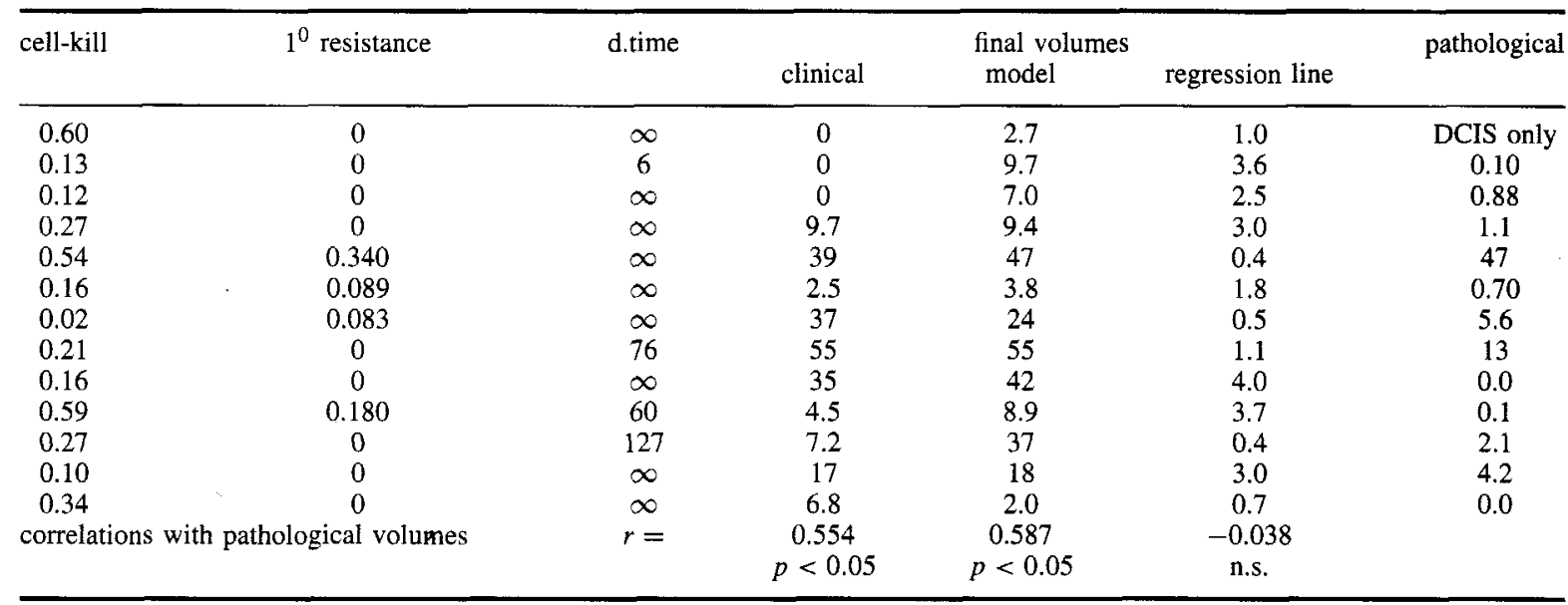

(Reproduced with modification from (Cameron et al. 1996) with permission) 
(which could be available $4-8$ weeks earlier), is no less accurate than the final clinical measurements taken just before surgery !

In order to test the advance this model makes over the simpler regression model, linear regression lines were fitted to the (logs of the) tumour volumes (as in (Forrest et al 1986; Johnson et al 1995)). It can be seen in table 1 that this approach is less accurate than both the clinical tumour measurements and the model predicted volumes, confirming it to be an inferior approach in the response assessment of primary breast cancer.

The same model was also applied to the tumour volumes of patients who had been treated in Edinburgh as part of the clinical study of pre-operative systemic therapy (vide supra), and a further cohort of women treated on a similar protocol as part of a subsequent randomised study comparing this pre-operative approach with conventional treatment consisting of surgery followed by adjuvant systemic therapy (Forouhi et al 1995). This combined data set of 63 women provides further evidence of this model to successfully model the response of breast cancer; in particular the detection of biological resistance to therapy. Patients that were modelled as having a primary breast cancer containing in excess of $8 \%$ initially resistant tumour had a worse prognosis, as shown in Figure 5. As for the patients with locally advanced breast cancer, all those with a pathological complete response had a $0 \%$ resistant term in the model. Finally, as was seen in the Priore study (Priore, 1966), using a model one can occasionally identify patients who appear to demonstrate tumour re-growth during therapy, although it would not be apparent from the actual tumour measurements; an example is given in Figure 6.

Why this model appears to be better than the regression line for predicting tumour regression can only be surmised, but since it is the proportion of resistant cells that provides information on a

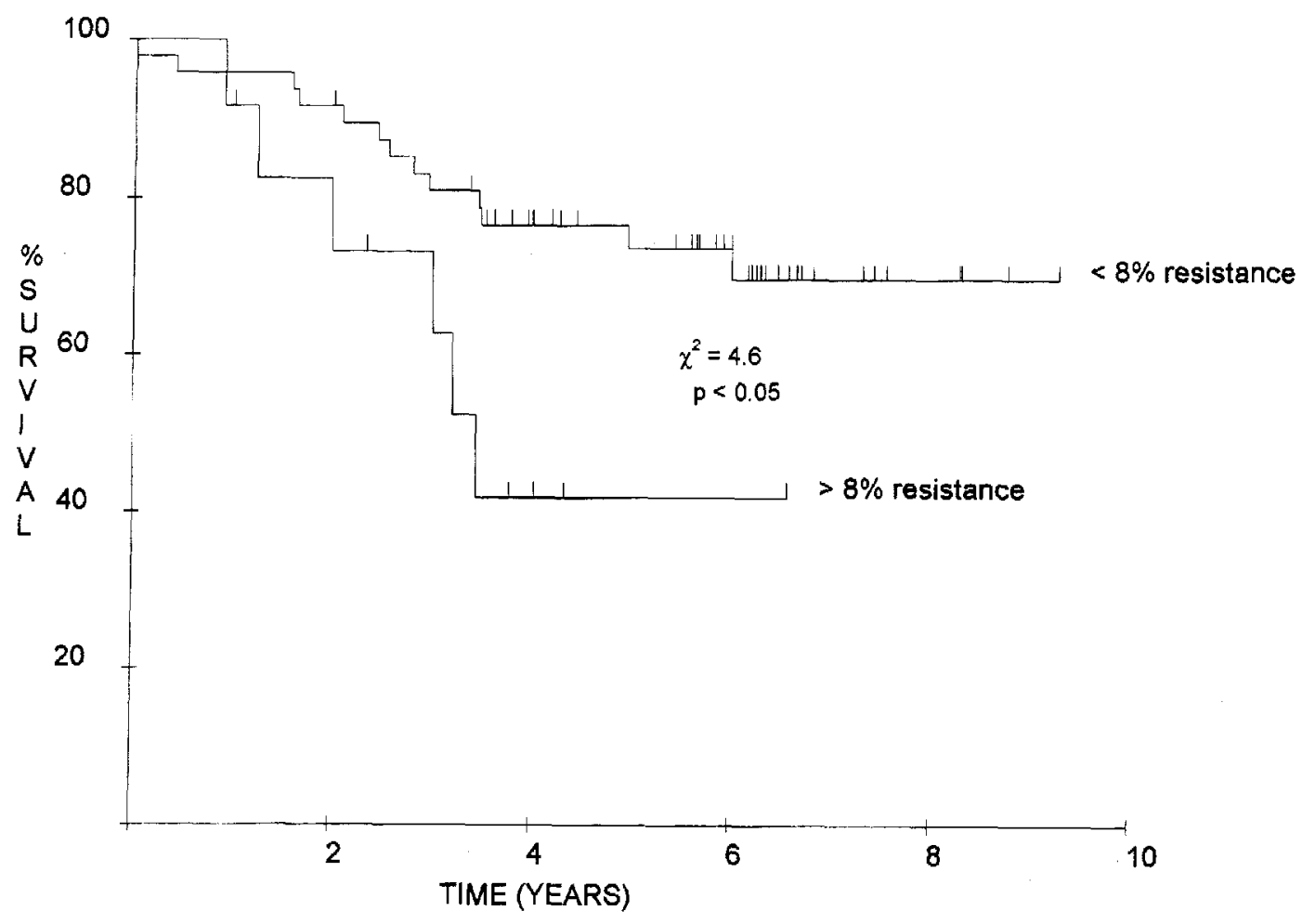

FIGURE 5 Kaplan-Meier survival for operable breast cancers, differentiated by the model-predicted resistance fraction of the tumour. Vertical lines represent censored patients. 


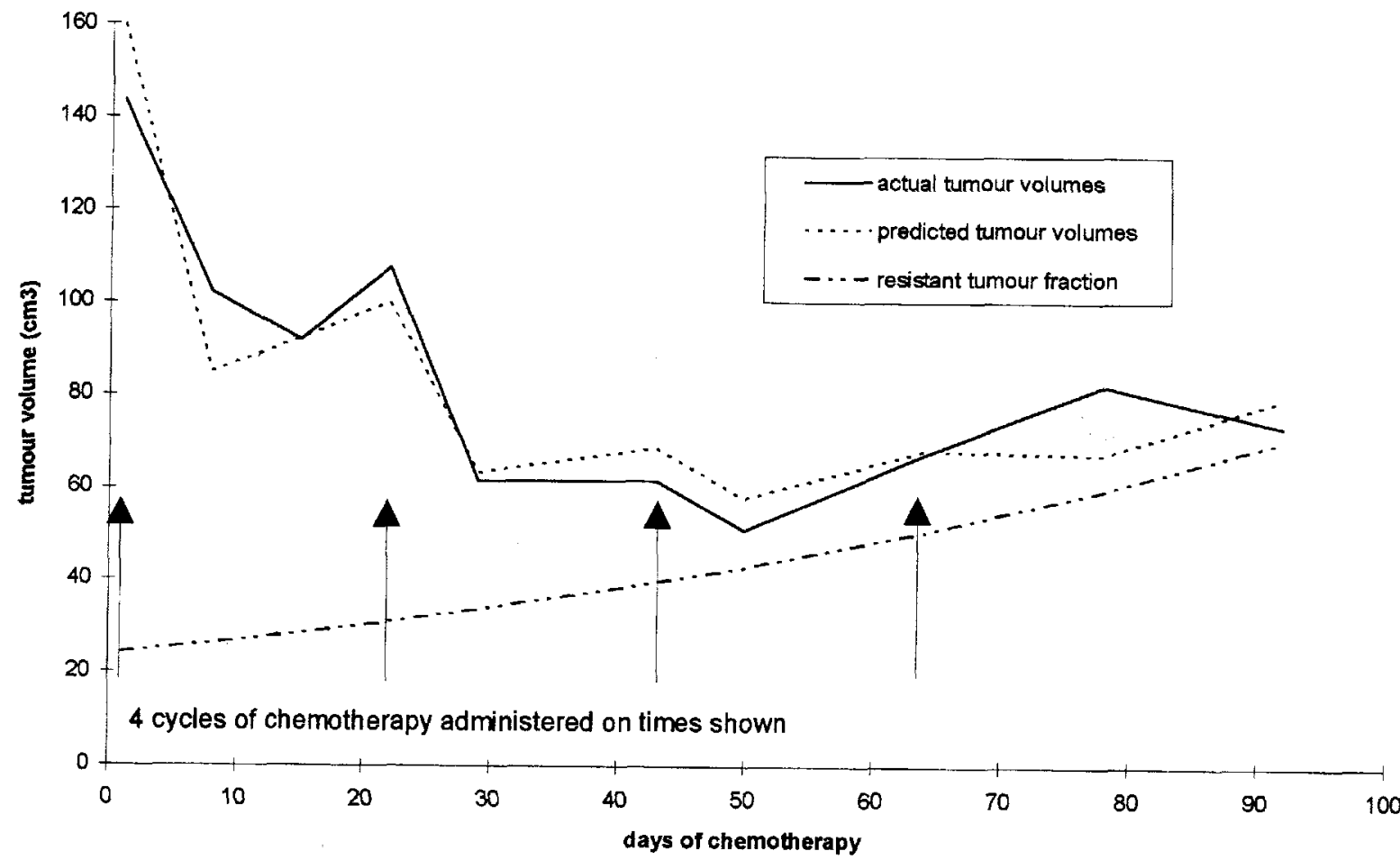

FIGURE 6 An example of the model detecting tumour regrowth for a pre-operatively treated early breast cancer.

patient's medium-term survival, one can surmise that the inclusion of this component in the model is critical to its ability to model tumour biology.

\section{GOMPERTZIAN GROWTH}

During the 1960 's it was realised that human tumours when xenografted into laboratory animals did not grow exponentially throughout their lifespan. In a seminal pair of papers, Laird showed clearly that the growth of such tumours is well modeled by Gompertzian growth, which describes exponential growth at each time point, but with continual deceleration and a maximum volume (Laird, 1965; Laird, 1964):

$$
W=W_{0} \exp \left(\frac{A}{\alpha}(1-\exp (-\alpha t))\right)
$$

(where $\boldsymbol{W}_{0}$ is the initial tumour size, $\boldsymbol{W}$ is the size at time t, and $\boldsymbol{A}$ and $\alpha$ are constants, such that the initial growth rate is $\boldsymbol{A}$, and $\alpha$ is the constant describing the rate of decay of the instantaneous growth rate).

Norton later demonstrated the ability of this function to predict subsequent xenograft growth (Norton et al 1976). The difference between this curve and exponential growth is that it is a form of bounded growth, with the maximum possible volume, $W_{\max }$, occurring $t=\infty$, equal to $W_{0} e^{(A / \alpha)}$. Brunton and Wheldon showed that in fact this maximum volume appears to be species-specific (Brunton et al 1978), and thus for a tumour grown in one animal there is potentially only one variable to describe its growth. The growth as described by this function is always exponential, but with an exponentially declining growth rate. There is thus a point of inflection, which occurs at $W_{\max } / e$, beyond which point no further doubling of the tumour occurs.

During the next decade, a number of papers examined the relevance of such a growth pattern to clinical tumours, and although some controversy was generated, many would hold to 
the view espoused that clinical tumours grow in a Gompertzian manner. The details of the arguments are not relevant, but certain points need to be made, since Gompertzian growth has not been used in any published model of the response of breast cancer to therapy, and might in fact not be the best description of human tumour growth.

The paper (Norton, 1988) that argued for Gompertzian growth of clinical breast cancer was actually a reply to a previous paper arguing that breast cancer grew intermittently, in Gompertzian manner, with interim periods of dormancy (Speer et al 1984). Norton showed very clearly that the same data sets could be fitted by Gompertzian growth without the necessity for any periods of dormancy, although there is later work suggesting that tumour dormancy might be a real phenomenon (Demicheli et al 1996; Demicheli et al 1994). However only one of the three sets of data employed was primary tumour growth data, and in that series (Heuser et al 1979) there were only 23 tumours with sufficient data. Furthermore, for that data set, and the natural history data set published by Bloom (Bloom et al 1962), it was found that $W_{\max }=$ $3.1^{*} 10^{12}$ cells, and that $\alpha$ ( $b$ in the paper) was lognormally distributed about a mean of $\log _{e}(-2.9)$. The inflection point occurs at a volume of $1.14^{*} 10^{12}$ cells, which is always greater than the lethal tumour cell burden of $10^{12}$ cells! For the fit of Heuser's data (Heuser et al 1979), it was reported that they all fitted within the lower range of values of $\alpha$ ( $b$ in the paper), and for the same $W_{\max }$. However the maximum volume observed on the mammograms was a tumour measuring $80 \times 90 \mathrm{~mm}$, corresponding to about $0.3^{*} 10^{12}$ cells, again before the inflection point.

This point is further illustrated by a small study on testicular tumour lung metastases (Demicheli, 1980). The largest observed diameter was $69 \mathrm{~mm}$, corresponding to $0.17^{*} 10^{12}$ cells. Using the published values of $A / \alpha$ ( $a_{0}$ in the paper) and $\alpha$ ( $b$ in the paper), the $W_{\max }$ for this tumour was $9.25^{*} 10^{10}$ cells. The only other tumour with a maximum volume (again assuming a sphere) greater than the inflection point was for patient number 8 , with a maximum metastasis volume of $1.4^{*} 10^{10}$ and an inflection volume of $1.1^{*} 10^{10}$ cells. Hence the currently accepted dogma that clinical tumour growth is Gompertzian is based primarily on data before the inflection point. This implies that the main feature that differentiates the Gompertz and logistic growth functions, namely the earlier inflection point and long period of time with significant growth deceleration that occurs with the former, is rarely spanned by the available clinical data, and only inferred from the fact that a Gompertzian curve has been found to fit. Hence if other curves could fit as well, the conclusions drawn from fitting Gompertzian curves (such as when the initial tumour cell appeared, and the implication for treatment schedules that tumours grow much faster when sub-clinical (Norton, 1993)) need to be reconsidered.

Furthermore two contrary views also need to be discussed. In a retrospective study of a large series of primary breast cancers, seen at the MD Anderson over 35 years, modelled with the assumption that the probability of clinical detection was proportional to the tumour size, the authors found no evidence for bounded growth of any form, with exponential growth (with up to 50-fold variation in growth rates) adequately describing the tumour sizes at presentation (Brown et al 1984). The other, more recent study that challenges the Gompertz model was based on the tumour volume as detected in patients with sequential mammograms. Spratt (Spratt et al 1993) found that the closest fits of the data were obtained with a logistic function, with $N=1 / 4$ giving the best fit:

$$
S=S_{\infty}\left(1+\frac{1}{\exp ((b t+c) / 4)}\right)^{-4}
$$

(where $S$ is the tumour volume at time $t, S_{\infty}$ is the maximum volume, and $\boldsymbol{b}$ and $\boldsymbol{c}$ are parameters, such that $b$ describes the rate of decceleration, and $c$ is defined in terms of $\boldsymbol{S}_{0}$ and $\boldsymbol{S}_{\infty}$.)

Although the data could be fitted with a Gompertzian function, this was with an $8 \%$ increase in mean-squared error, whereas an exponential growth model gave rise to a $17 \%$ increase in 
error. The significant difference between logistic and Gompertz curves is that the former only produce significant growth deceleration at volumes close to the maximum (and beyond the range of accurate mammography).

To illustrate how a limited data-set, such as that provided by Heuser, can in fact be fitted by different curves, Figures $7 \mathrm{a}$ and $7 \mathrm{~b}$ illustrate respectively the fastest and slowest growing tumours in Heuser's dataset (Heuser et al 1979). A Gompertz curve, a simple exponential and a logistic curve (using $N=1 / 4$ after Spratt (Spratt et al 1993)) have all been drawn to exactly intersect with the two published mammographic volumes, and for the latter two functions, using the same maximum volume as Norton $\left(3.1^{*} 10^{12}\right.$ cells). It can be clearly seen that there is little difference over the clinically relevant range for the logistic and Gompertz curves, whereas the implications for the growth curves well away from the clinically available data are very different for the exponential curve.

In conclusion therefore, although the Gompertz function does appear to describe the growth of human tumours as xenografts and can describe clinical disease, the data do not confirm that it to be the most accurate model for primary (nonmetastatic) breast cancer. However it is relevant to note that the Gompertz function has been successfully applied as a model of the behaviour of another human malignancy, multiple myeloma, whose hallmark is the production of a monoclonal myeloma protein the concentration of which can be measured in serum (Sullivan et al 1972). Tumour volume cannot be determined in this condition, but the total body production of the myeloma protein can be deduced, and used as an accurate surrogate for the total tumour burden. In a study of approximately 30 patients, accurate prediction of the tumour regression with treatment was possible, and better classification of partial responders with concomitant survival benefit was achieved than had been possible simply on the basis of the serum concentration of the myeloma protein. This study also employed "log-cell kill", in which a given dose of drug kills a fixed proportion of the tumour cells, as demonstrated for leukemia cells in vitro (Skipper, 1978). However it should be mentioned en passant, that even for the patients in this study, the majority had initial tumour volumes before the point of inflection of their Gompertz growth curve.

\section{CONCLUSION}

Firstly it needs to be restated that although the laboratory data on tumour growth is accurately modelled by a Gompertzian growth curve, the published clinical data is less supportive, particularly since the volumes used are mostly before the inflection point of the Gompertz function. It may be that other bounded growth functions such as the logistic will provide a better description of human tumour growth, as one study suggests. Therefore the fact that the currently published models of tumour response to therapy are not based on Gompertzian growth does not undermine them, particularly since they use a growth function to model re-growth after therapy, when the volume could be below the clinically detectable threshold. Furthermore post-treatment regrowth is, because of problems in experimental design, a poorly studied phenomenon.

Confirmation as to what is the best model of clinical tumour growth can only come when there is a larger body of data to which the various curves can be fitted. There are however two major stumbling blocks to collecting the appropriate data. In the first place it is unusual to be able to watch tumours grow without therapeutic intervention, particularly in breast cancer for which it would often be unethical not to offer treatment. Furthermore tumour volume measurement is difficult with metastatic disease, and therefore the bulk of clinical data comes from disease within the breast or lung fields. But the most pressing need is for more data on tumour volumes earlier in the course of the disease. and the difficulty in collecting this is the same as that faced by screening programmes, namely that it is hard to identify tumours much below $10^{8}-10^{9}$ cells (corresponding to volumes of the order of a cubic centimetre). Figures $7 a$ and $b$ suggest that 
clear separation of the logistic, exponential and gompertz curves only occurs below $10^{7}$ cells - and if clinicians could measure the disease at that stage, then the prospects for early surgical cure might be much improved!

The models presented all provide some degree of accuracy of tumour growth, but that written by Gregory is the only one that has been shown to predict subsequent clinical behaviour, such as the pathological tumour volume or the identification of patients with a worse prognosis. That it is not perfect is no surprise - the search for biological markers that provide good prognostic discrimination in breast cancer has not been a great deal more successful. Clearly further improvements are necessary, but important refinement of these models may only come when they can incorporate the increased understanding of the cellular mechanisms of celldeath and re-growth in response to therapy.

\section{APPENDIX 1}

\section{W. Gregory's Model of Exponential Cell Growth and Log-Cell Kill}

With the assumptions that all growth is exponential, and that the same proportion $k$ of the sensitive tumour is killed by each cycle of therapy, the tumour volumes $\left(X_{0}, X_{1}, X_{2}, \ldots, X_{n}\right)$ will be described by the equation:

$$
X_{i}=\frac{1-a-\left(1-a^{i}\right) k_{0}}{1-a} X_{0} \exp \left(\alpha t_{i}\right)
$$

where $a=(1-k)$ and $k_{0}=k\left(1-R_{0}\right) . R_{0}$ is the proportion of the tumour initially resistant, $\alpha$ is the growth rate, $t_{i}$ is the time measured in days between the first treatment and treatment cycle $i+1$, and $i$ is the treatment cycle number itself. Then from equation (1)

$$
\log X_{i}=\log \left[\frac{1-a-\left(1-a^{i}\right) k_{0}}{1-a}\right]+\log X_{0}+\alpha t
$$

Let the actual tumour volumes be $V_{0}, V_{1}, \ldots, V_{n}$. We have assumed that these are lognormally distributed about the true volumes with some constant standard deviation $\sigma$ (equivalent to the assumption that the tumours are measured on each occasion with the same percentage error).

Therefore the likelihood $L$ of the (log of) these volumes under the model is:

$$
\begin{aligned}
L\left(\log V_{0}, \log V_{1}, \ldots, \log V_{n}\right)=N\left(\log V_{0}, \log X_{0}, \sigma\right) & \cdot N\left(\log V_{1}, \log X_{1}, \sigma\right) \ldots N\left(\log V_{n}, \log X_{n}, \sigma\right) \\
= & \Pi_{i=0}^{n} N\left(\log V_{i}, \log X_{i}, \sigma\right)
\end{aligned}
$$

where $\mathrm{N}(\mathrm{x}, \mathrm{m}, \mathrm{s})$ is the value of a normal distribution with mean $\mathrm{m}$ and variance $s^{2}$ at $\mathrm{X}$.

Thus

$$
\begin{aligned}
& \log L=\sum_{i=0}^{n} \log N\left(\log V_{i}, \log X_{i}, \sigma\right) \\
& \text { Now } N(x, \mu, \sigma)=\frac{1}{\sigma \sqrt{2 \pi}} \exp \left[\frac{-(\mu-x)^{2}}{2 \sigma^{2}}\right] \\
& \text { Hence } \log L=\sum_{i=0}^{n} \log \left[\frac{1}{\sigma \sqrt{2 \pi}}\right. \\
& \left.\quad \times \exp \left[\frac{-\left(\log X_{i}-\log V_{i}\right)^{2}}{2 \sigma^{2}}\right]\right]
\end{aligned}
$$

The maximum likelihood estimates (MLRs) for $X_{0}, k, R_{0}, \alpha$ and $\sigma$ (i.e. the values of these parameters which produce the closest fit between the model's predictions and the data) can then be determined by maximising $L$ from equation (2). This can be achieved by differentiating $\log L$ with respect to each of the parameters $X_{0}, k, R_{0}, \alpha$ and $\sigma$ and maximising $\log L$ based on the values of these derivatives using a semi-Newtonian algorithm (reproduced from (Cameron et al 1996) with permission).

\section{References}

Bloom, H., Richardson, M. and Harries, B. (1962) Natural history of untreated breast cancer (1804-1933); comparison of untreated and treated cases according to histological grade of malignancy, British Medical Joumal, 2, 213-221.

Brown, B. W., Atkinson, E. N., Bartoszynski, R., Thompson, J. R. and Montague, E. D. (1984) Estimation of Human Tumour Growth Rate From Distribution of tumour Size at Detection, Journal of the National Cancer Institute, 72, 31-38. 
Brunton, G. F. and Wheldon, T. E. (1978) Characteristic species dependent growth patterns of mammalian neoplasms, Cell Tissue kinet, 11, 161-175.

Calais, G., Descamps, P., Chapet, S., Turgeon, V., ReynaudBougnoux, A., Lemarié, E., Fignon, A., Body, G., Bougnoux, P., Lansac, J. and Le Floch, O. (1993) Primary chemotherapy and radiosurgical breast-conserving treatment for patients with locally advanced operable breast cancer, International Journal of Radiaton Oncology Biology and Physics, 26, 37-42.

Cameron, D. A., Anderson, E. D., Levack, P., Forrest, A. P. M., Leonard, R. C. F. and Chetty, U. (1995) Long-term follow-up of neo-adjuvant therapy in operable breast cancer-an update of the original Edinburgh series, The Breast, 4, 240-(abst 48), (Abstract).

Cameron, D. A., Gregory, W. M., Bowman, A. and Leonard, R. C. F. (1996) Mathematical modelling of tumour response in primary breast cancer, British Journal of Cancer, 73, 1409-1416.

Cameron, D. A., Anderson, E. D. C., Levack, P., Hawkins, R. A., Anderson, T. J., Leonard, R. C. F., Forrest, A. P. M. and Chetty, U. (1997) Primary systemic therapy for operable breast cancer - 10-year survival data following chemotherapy and hormone therapy, British Journal of Cancer, (in press).

Chevillard, S., Pouillart, P., Beldjord, G., Asselain, B., Beuzeboc, P., Magdelenat, H. and Viehl, P. (1996) Sequential assessment of multidrug-resistance phenotype and measurement of $S$-phase fraction as predictive markers of brest-cancer response to neoadjuvant chemotherapy, Cancer, 77, 292-300.

Clayton, F. (1991) Pathologic correlates of survival in 378 lymph node-negative infiltrating ductal breast carcinomas, Cancer, 68, $1309-1317$.

Collins, V. P., Loeffler, R. K. and Tivey, H. (1956) Observations on growth rates of human tumours, Am J Roentgenol, 76, $988-1000$

Demicheli, R. (1980) Growth of Testiculat neoplasm Lung Metastases: Tumor-Specific Relation Between Two Gompertzian Parameters, European Journal of Cancer, 16, 1603-1608.

Demicheli, R., Terenziani, M., Valagussa, P., Moliterni, A., Zambetti, M. and Bonadonna, G. (1994) Local Recurrences Following Mastectomy: Support for the Concept of Tumor Dormancy, Journal of the National Cancer Institute, 86, 45-48.

Demicheli, R., Abbattista, A., Miceli, R., Valagussa, P. and Bonadonna, G. (1996) Time distribution of the recurrence risk for breast cancer patients undergoing mastectomy: further support about the concept of tumour dormancy, Breast Cancer Research and Treatment, 41, 177-185.

Early Breast Cancer Trialists Collaborative Group (1992) Systemic treatment of early breast cancer by hormonal, systemic or immune therapy: 133 randomised trials involving 31,000 recurrences and 24,000 deaths among 75,000 women, Lancet, $339,1-14$ and $71-85$.

Epstein, R. (1996) Treating breast cancer before surgery, British Medical Journal, 313, 1345-1346.

Fisher, B., Gunduz, N. and Saffer, E. A. (1983) Influence of the interval between primary tumour removal and chemotherapy on kinetics and growth of metastases, Cancer Research, 43, $1488-1492$.

Forouhi, P., Dixon, J. M., Leonard, R. C. F. and Chetty, U. (1995) Propspective randomized study of surgical morbidity following primary systemic therapy for breast-cancer, British Journal of Surgery, 82, 79-82.

Forrest, A. P. M., Levack, P. A., Chetty, U., Hawkins, R. A., Miller, W. R., Smyth, J. F. and Anderson, T. J. (1986) A human tumour model, Lancet, 2, 840-842.
Gardin, G., Alama, A., Rosso, R., Campora, E., Repetto, L.. Pronzato, P., Merlini, L., Naso, C., Camoriano, A., Meazza, R., Barbieri, F., Baldini, E., Giannessi, P. G. and Conte. P. F. (1994) Relationship of variations in tumour cell kinetics induced by primary chemotherapy to tumour regression and prognosis in locally advanced breast cancer, Breast Cancer Research and Treatment, 32, 311-318.

Gasparini, G., Boracchi, P., Verderio, P. and Bevilacqua, P. (1994) Cell kinetics in human breast cancer: comparison between the prognostic value of the cytofluorimetric S-phase fraction and that of the antibodies to Ki-67 and PCNA antigens detected by immunohistochemistry, International Journal of Cancer, 57, 822-829.

Gompertz, B. (1825) On the nature of the function expressive of the law of human mortality, and on a new method of determining the value of life contingencies, Phil Trans Roy Soc, 513-585.

Gregory, W. M., Reznek, R. H., Hallet, M. and Slevin, M. L. (1990) Using mathematical models to estimate drug resistance and treatment efficacy via CT scan measurements of tumour volume, British Journal of Cancer, 62, 671-675.

Harris, A. L. and Hochhauser, D. (1992) Mechanisms of multidrug resistance in cancer treatment, Acta Oncologica, 31, $205-213$.

Hayward, J. L., Carbonne, P. P., Heuson, J. C., Kumaoka, S., Segaloff, A. and Rubens, R. D. (1977) Assessment of response to therapy in advanced breast cancer, Cancer, 39, $1289-1294$.

Henderson, I. C. (1995) Paradigmatic shifts in the management of breast cancer, New England Journal of Medicine, 332, 951-952.

Heuser, L., Spratt, J. S. and Polk, H. C. (1979) Growth rates of primary breast cancers, Cancer, 43, 1888-1894.

Ingleby, H., Moore, L. and Gershon-Cohen, J. (1958) A Roentgenographic study of growth rate of 6 "early" cancers of the breast, Cancer, 11, 726-730.

Jacquillat, C., Weil, M., Baillet, F., Borel, C., Auclerc, G. de Maublanc, M. A., Housset, M., Forget, G., Thill, L., Soubrane, C. and Khayat, D. (1990) Results of neoadjuvant chemotherapy and radiation therapy in the breast-conserving treatment of 250 patients with all stages of infiltrative breast cancer, Cancer, 66, 119-129.

Johnson, A. E., Bennett, M. H., Cox, S. J., Cheung, C. W. D. and Sales, J. E. L. (1995) The management of individual breast cancers, The Breast, 4, 100-111.

Laird, A. K. (1964) Dynamics of tumor growth, British Journal of Cancer, 18, 490-502.

Laird, A. K. (1965) Dynamics of tumour growth: comparison of growth rates and extrapolation of growth curve to one cell, British Journal of Cancer, 19, 278-291.

Mauriac, L., Durand, M., Avril, A. and Dilhuydy, J.-M. (1991) Effects of primary chemotherapy in conservative treatment of breast cancer patients with operable tumours larger than $3 \mathrm{~cm}$, Annals of Oncology, 2, 347-354.

Norton, L., Simon, R., Brereton, H. D. and Bogden, A. E. (1976) Predicting the course of Gompertzian growth, Nature, 264, 542-545

Norton, L. (1988) A Gompertzian Model of Human Breast Cancer Growth, Cancer Research, 48, 7067-7071.

Norton, L. (1993) Kinetic Concepts in the Treatment of Breast Cancer. In: Recent Results in Cancer Research, SpringerVerlag Berlin, Heidelberg, pp. 1-6.

Powles, T. J., Hickish, T. F., Makris, A., Ashley, S. E., O'Brien, M. E. R., Tidy, V. A., Casey, S., Nash, A. G., Sacks, N.. Cosgrove, D., MacVicar, D., Fernando, I. and Ford, H. T. 
(1995) Randomized trial of chemoendocrine therapy started before or after surgery for treatment of primary breast cancer, Journal of Clinical Oncology, 13, 547-552.

Priore, R. L. (1966) Using a mathematical model in the evaluation of human tumor response to chemotherapy, Journal of the National Cancer Institute, 37, 635-647.

Remvikos, Y., Jouve, M., Beuzeboc, P., Viehl, P., Magdelenat, H. and Puoillart, P. (1993a) Cell cycle modifications of breast cancers during neoadjuvant chemotherapy: A flow cytometry study on fine needle aspirates, European Journal of Cancer, 29, 1843-1848.

Remvikos, Y., Mosseri, V., Zajdela, A., Fourquet, A., Durand, J. C., Pouillart, P. and Magdelenat, H. (1993b) Prognostic value of the S-phase fraction of breast cancers treated by primary radiotherapy or neoadjuvant chemotherapy, Annals of the New York Academy of Science, 698, 193-203.

Scholl, S. M., Fourquet, A., Asselain, B., Pierga, J. Y., Vicoq, J. R., Durand, J. C., Dorval, T., Palangié), T., Jouve, M., Beuzeboc, P., Garcio-Giratl, E., Salmon, R. J., de la Rochefordière, A., Campana, F. and Pouillart, P. (1994) Neoadjuvant versus adjuvant chemotherapy in premenopausal patients with tumours considered too large for breast conserving surgery: preliminary results of a randomised trial: S6, European Journal of Cancer, 30A, 645-652.

Scholl, S. M., Asselain, B., Beuzeboc, P., Pierga, J. Y., Dorval, T., Garcia-Giralt, E., Jouve, M., Palangiê, T., Fourquet, A., Durand, J. C. and Pouillart, P. (1995) Neoadjuvant versus adjuvant chemotherapy in premenopausal patients with tumours considered too large for conserving surgery: an update, Anti-Cancer Drugs, 6(2), 69-(abst P48).

Scholl, S. M., Pierga, J. Y., Asselain, B., Buezeboc, P., Dorval, T., Garcia-Giralt, E., Jouve, M., Palangié, T., Remvikos, Y., Durand, J. C., Fourquet, A. and Pouillart, P. (1996) Breast tumour response to primary chemotherapy predicts local and distant control as well as survival, European Journal of Cancer, 31A, 1969-1975.

Schwartz, M. (1961) A biomathematical approach to clinical tumour growth, Cancer, 14, 1272-1294.

Semiglazov, V. F., Topuzov, E. E., Bavli, J. L., Moiseyenko, V. M., Ivanova, O. A., Seleznev, I. K., Orlov, A. A., Barash. N. Y., Golubeva, O. M. and Chepic, O. F. (1994) Primary (neoadjuvant) chemotherapy and radiotherapy compared with primary radiotherapy alone in stage llb-llla breast cancer. Annals of Oncology, 5, 591-595.

Skehan, P. (1984) Cell growth, tissue neogenesis and neoplastic formation. In Skehan, P., Friedman, S. J. (eds.) "Growth Cancer and the Cell Cycle." The Humana Press, Clifton, NJ. 323-345.

Skipper, H. E. (1978) "Reasons for Success and Failure in the treatment of murine Leukemias with the drugs now employed in treating human leukemias, 1, University Microfilms International, Ann Arbor.

Speer, J. F., Petrosky, V. E., Retsky, M. W. and Wardwell. R. H. (1984) A Stochastic Numerical Model of breast Cancer Growth That Simulates Clinical Data, Cancer Research, 44, 4124-4130.

Spratt, J. A., von Fournier, D., Spratt, J. S. and Weber, E. E. (1993) Decelerating Growth and Human Breast Cancer, Cancer, 71, 2013-2019.

Sullivan, P. W. and Salmon, S. E. (1972) Kinetics of Tumor Growth and Regression in IgG Multiple Myeloma, The Journal of Clinical Investigation, 51, 1697-1708.

Thomlinson, R. H. (1982) Measurement and management of carcinoma of the breast, Clinical Radiology, 33, 481-493.

Verhulst, P. F. (1838) Notice sur la loi que la population suit dans son accroissement, Corr Math Phys, 10, 113-121.

Winsor, C. P. (1932) The Gompertz curve as a growth curve. Proc Nat Acad Science, 18, $1-8$. 


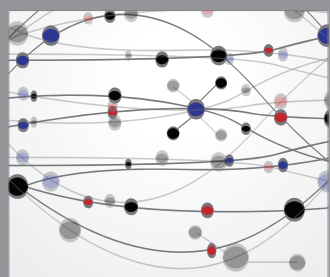

The Scientific World Journal
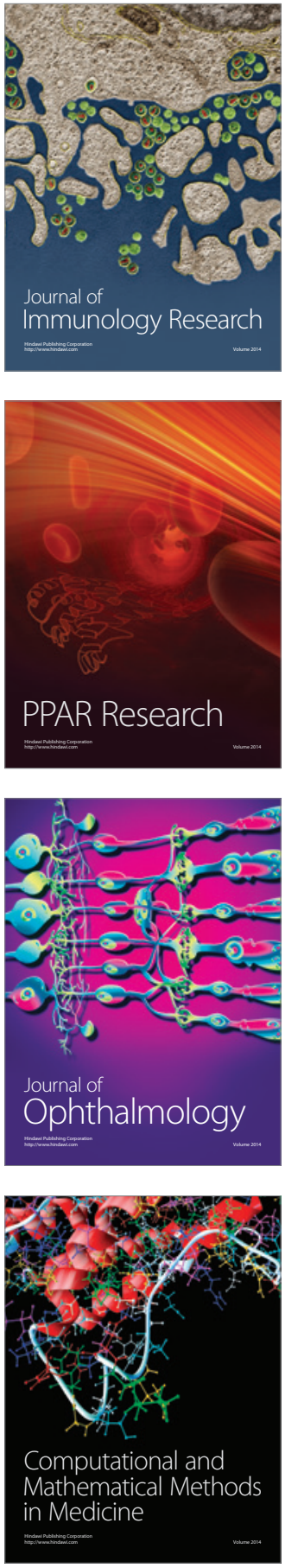

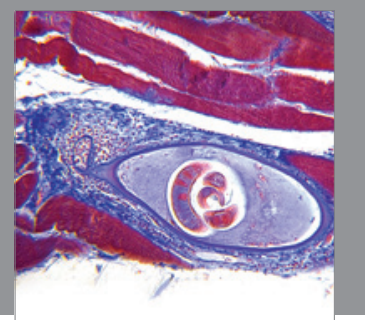

Gastroenterology

Research and Practice
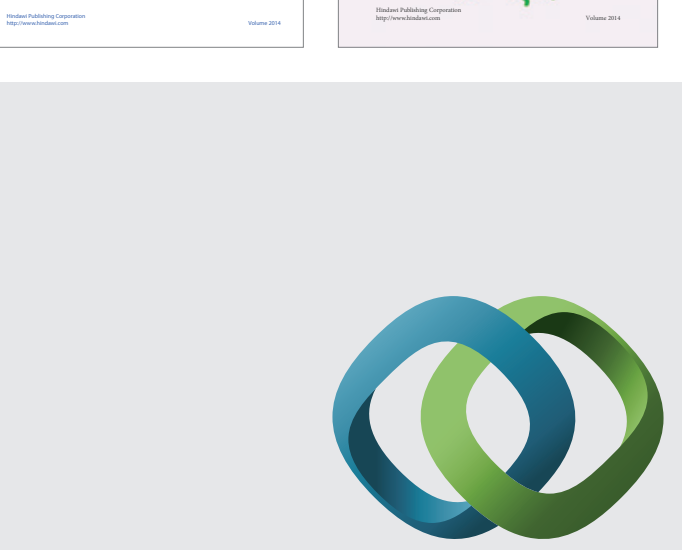

\section{Hindawi}

Submit your manuscripts at

http://www.hindawi.com
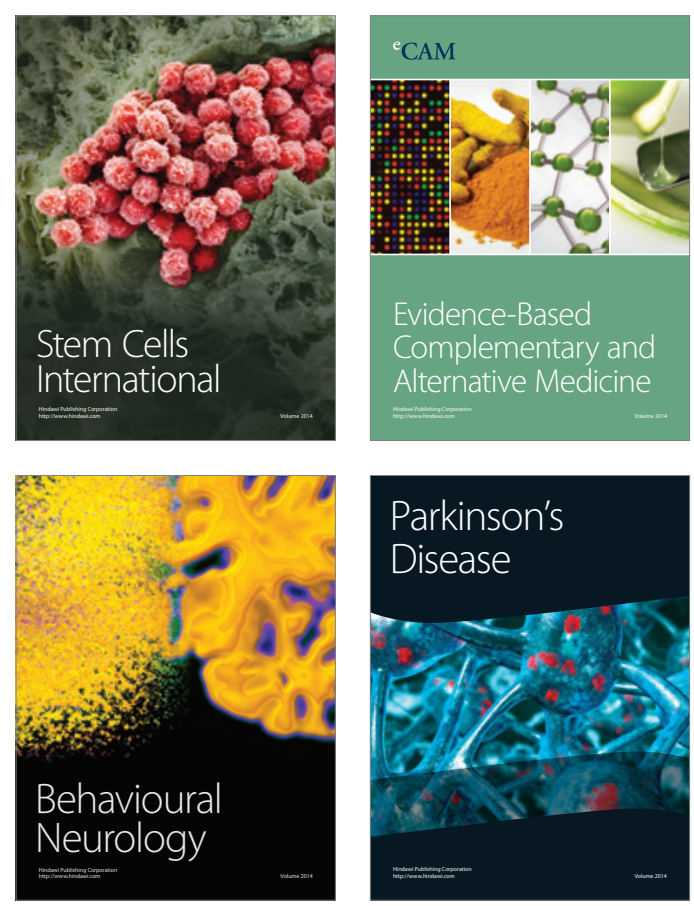

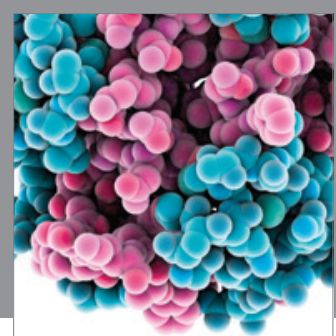

Journal of
Diabetes Research

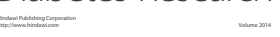

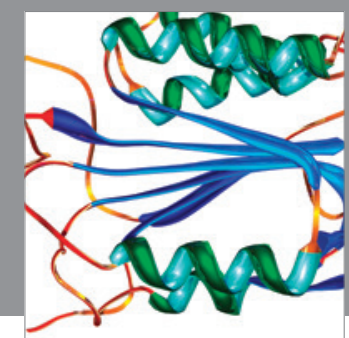

Disease Markers
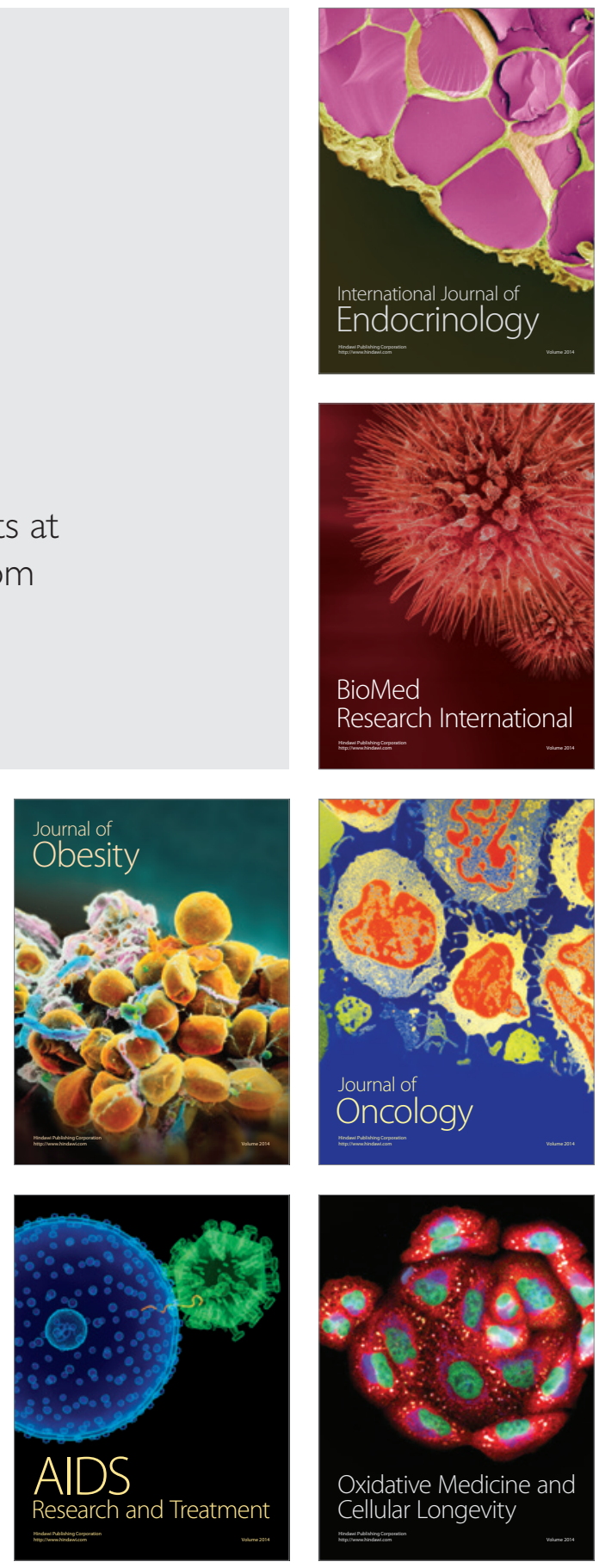